\title{
Low-Protein, Hypocaloric Nutrition with Glutamine versus Full-Feeding in the Acute Phase in ICU Patients with Severe Traumatic Brain Injury
}

This article was published in the following Dove Press journal:

Neuropsychiatric Disease and Treatment

\author{
Weichuan Xiong $\mathbb{( D}^{1,2}$ \\ Kejian Qian ${ }^{2}$ \\ 'Department of Critical Care Medicine, \\ The Third Affiliated Hospital of Nanchang \\ University, Nanchang, 330006, People's \\ Republic of China; ${ }^{2}$ Department of \\ Critical Care Medicine, The First \\ Affiliated Hospital of Nanchang \\ University, Nanchang, 330006, People's \\ Republic of China
}

Objective: To investigate the 28-day mortality, the length of ICU stay, days in the hospital, days of ventilator use, adverse events, and nosocomial infection events of low-protein, hypocaloric nutrition with glutamine in the first 7 days of the intensive care unit (ICU) patients with severe traumatic brain injury (STBI).

Patients and Methods: A total of 53 patients diagnosed with STBI enrolled from the third affiliated hospital of Nanchang University (Nanchang, China), from January 2019 to July 2020, were divided into two groups. We performed a randomized prospective controlled trial. The intervention group $(\mathrm{n}=27)$ was nutritional supported (intestinal or parenteral) with a caloric capacity of $20-40 \%$ of European Conference on Clinical Nutrition and Metabolism (ESPEN) recommendations; specifically, low-protein intake was $0.5-0.7 \mathrm{~g} / \mathrm{kg}$ per day (containing the amount of alanyl-glutamine), glutamine was $0.3 \mathrm{~g} / \mathrm{kg}$ per day, and the intervention treatment lasted for 7 days. The control group $(n=26)$ was nutritionally supported with a caloric capacity of $70-100 \%$ of ESPEN recommendations, and the protein intake was $1.2-1.7 \mathrm{~g} / \mathrm{kg}$ per day. The primary endpoint was 28 -day mortality. Secondary endpoints were the length of ICU stay, days in the hospital, days of ventilator use, adverse events and nosocomial infection events.

Results: There were no differences in baseline characteristics between groups. Survival curve analysis using the Kaplan-Meier method revealed no significant difference in 28-day mortality between the two groups $(P=0.31)$ while adverse events $\left(\chi^{2}=5.853, P=0.016\right)$, nosocomial infection rate $\left(\chi^{2}=4.316, P=0.038\right)$, the length of ICU stay $(\mathrm{t}=-2.617$, $P=0.012)$, hospitalization time $(\mathrm{t}=-2.169, P=0.036)$, and days of ventilator use $(\mathrm{t}=-2.144$, $P=0.037$ ) of patients in the intervention group were significantly lower than those in the control group.

Conclusion: Low-protein, hypocaloric nutrition with glutamine did not show different outcomes in 28-day mortality compared to full-feeding nutritional support in the ICU patients with STBI. However, low-protein, hypocaloric nutrition with glutamine could provide a lower need for ICU time, hospitalization time, and ventilator time in the ICU patients with STBI.

Keywords: acute phase, severe traumatic brain injury, nutrition, low-protein, hypocaloric, glutamine

\section{Introduction}

Brain injury indicates a severe trauma caused by violence on the head. Its mortality rate has dropped by $50 \%$ in the past 20 years. One of the reasons for the gradual decrease in brain injury mortality is the continuous improvement of intensive care
Correspondence: Kejian Qian

Email kejianqian@sina.com
Neuropsychiatric Disease and Treatment 2021:17 703-710 
technology. ${ }^{1}$ In the treatment of patients with severe brain injury, nutritional support plays a vital role in improving the prognosis of patients with severe brain injury. Now everyone's consensus is that proper nutritional support is of great significance for the functional recovery of patients with severe brain injury. However, whether early nutrition can help reduce the abnormalities in the acute phase and improve the prognosis of patients is controversial, and has not been supported by the latest experiments in this field.

ICU clinicians often ask questions at this stage: Should patients with severe brain injury start nutritional support treatment in the early stage of the disease? Does adding glutamine help improve the prognosis? These questions have not been clearly answered by the latest ESPEN ${ }^{2}$ and the American Neurosurgery Guidelines. ${ }^{3}$

Therefore, these problems prompted us to explore whether the low-protein and low-calorie nutritional with glutamine in the acute phase is beneficial to improve the clinical outcome of patients with severe brain injury or not.

\section{Patients and Methods}

\section{Patients}

The study sample including 53 STBI patients was enrolled at the Department of intensive care unit (ICU) and Emergency intensive care unit (EICU) of the third affiliated hospital of Nanchang University (Nanchang, China), from January 2019 to July 2020. Patients meeting the inclusion criteria were randomly divided into an intervention group and a control group according to the random number. The randomization list is generated by a computer. We used opaque, sealed, and sequentially numbered envelopes to seal the random number. The feeding strategy cannot be designed in a double-blind manner due to the need to provide corresponding nutritional support. Only patients who completed 7 days of treatment were considered for analysis, and patients who did not end the follow-up period were excluded. The random number was returned to the sequence of patient replacement until the sample size was completed.

Inclusion criteria: (1) patients with non-surgical treatment with a GCS score of 4 to 8; (2) patients with no previous serious diseases of the blood system, respiratory system, endocrine system, cardiovascular system, and central nervous system, and no severe combined injury and skull base fracture; (3) all the selected cases were admitted to the hospital within 8 hours after the injury.
Exclusion criteria: (1) hemorrhagic shock, severe cardiopulmonary disease, children under 18 and elderly over 70 years old; (2) patients who had undergone surgery or died during the observation and treatment process (7 days).

The study was approved by the Ethics Committee of China Clinical Trial Center. This study was conducted in accordance with the Declaration of Helsinki. Written informed consent before enrollment in the study was provided by relatives. This trial has been registered in the Chinese Clinical Trial Registry, with the identifier of ChiCTR1800020106.

\section{Methods}

All patients were routinely provided with corresponding treatments such as sedation, hypothermia, muscle relaxants, dehydration, and anti-infection. According to ESPEN ${ }^{2} 2018$, the full feeding group (control group) and the permissive underfeeding group (intervention group) were determined.

The intervention group were nutritional supported (intestinal or parenteral) with a caloric capacity of $20-40 \%$ of European Conference on Clinical Nutrition and Metabolism (ESPEN) recommendations; specifically, low-protein intake was $0.5-0.7 \mathrm{~g} / \mathrm{kg}$ per day (containing the amount of glutamine), glutamine was $0.3 \mathrm{~g} / \mathrm{kg}$ per day, and the intervention treatment lasted for 7 days.

The control group was nutritionally supported with a caloric capacity of $70-100 \%$ of ESPEN recommendations, and the protein intake was $1.2-1.7 \mathrm{~g} / \mathrm{kg}$ per day, without glutamine.

The calorie and protein intake of the intervention group and the control group gradually returned to normal after the 8 th day. The nutritional route of the two groups of patients was first enteral nutrition, and the enteral nutrition given was full-fledged. In the control group, two bottles of amino acids were supplemented parenterally to achieve the protein required for the test.

\section{Testing Indexes}

The primary endpoint was 28-day mortality. Secondary endpoints were adverse events (diarrhea, gastric retention, refeeding syndrome), the length of ICU stay, days in the hospital, days of ventilator use, and nosocomial infection events.

\section{Statistical Analyses}

Sample size was calculated using PASS 15.0.5 software (NCSS, LLC. Kaysville, Utah, USA), The calculation of the sample size is based on the ability to change between groups (at least $80 \%$ of the power) during the 1 day of ICU 
hospitalization, and the power to simultaneously detect the $5 \%$ change in mortality during ICU hospitalization.

$\mathrm{R}$ open-source software (version 3.6.0) was used for statistical analysis. The Kaplan-Meier method was used for survival curve analysis. When the two groups of data are normally distributed data, $t$-test were used; Contingency Table $2 \times 2$ in size were analyzed with chi-square, Contingency Table $2 \times 5$ in size were analyzed with Fisher exact test when sparse data ( $b<5$ observations) were present. The Kaplan-Meier method was used to analyze the survival curve. $P$-value $<0.05$ is considered statistically significant.

\section{Results}

\section{Baseline Characteristics}

A total of 53 patients (male 37, female 16, age 22-69) with STBI were enrolled in this study and randomly divided into two groups. As presented in Table 1, there was no significant difference in gender, age, BMI score, APACHE II score, and GCS score between the two groups.

\section{Changes in Secondary Endpoints After Treatment in Both Groups}

Patients in the intervention group exhibited lower adverse events $(P=0.038$, Table 2$)$, lower probability of nosocomial infection $(P=0.016$, Table 2$)$, shorter ICU time $(P=0.012$, Figure 1$)$, shorter hospital stay $(P=0.036$, Figure 1), and longer time non-ventilator $(P=0.037$, Figure 1) compared to the control group.

\section{Comparison of the 28-Day Mortality Between the Two Groups}

The Kaplan-Meier method was used to analyze the survival curve, revealing that the ICU survival rate of the intervention group was slightly higher than that of the control group while there was no statistically significant difference $(P=0.31$, Figure 2$)$.

\section{Subgroup Analysis Based on GCS Score}

According to the GCS score, patients with 4 points and 5 points were combined into group I, and patients with 6 points, 7 points and 8 points were combined into group II. The survival rates of the intervention group and the control group on the 28th day were compared in group I and group II, respectively. As indicated by the GCS score in group II, the 28th-day survival rate of the intervention group was significantly higher than that of the control group, (Figure 3).

\section{Discussion}

Traditionally, it is recommended to provide adequate nutritional support in the acute phase of critical illness to reduce catabolism and prevent malnutrition. This is considered to be a window period. Early nutritional support can reduce the severity of the disease and accelerate the recovery from inflammatory syndrome (SIRS). The nutritional treatment provided by clinicians during this time is a non-nutritional benefit. ${ }^{4}$ In the past ten years, experts in critical care medicine believed that complete starvation is essential for preserving autophagy. If feeding is provided

Table I Baseline Characteristics of the Study Cohort

\begin{tabular}{|c|c|c|c|c|}
\hline Characteristics & Intervention Group $(n=27)$ & Control Group $(n=26)$ & t $/ \chi^{2}$ value & $P$-value \\
\hline Age mean $(S D)^{a}$ & $49.44(14.94)$ & $48.3 I(\mid 0.4 I)$ & $\mathrm{t}=0.322$ & $P=0.749$ \\
\hline Male $n(\%)^{b}$ & $20(74.07)$ & $17(65.38)$ & $\chi^{2}=0.475$ & $P=0.491$ \\
\hline $\mathrm{BMI}$ mean $(\mathrm{SD})^{\mathrm{a}}$ & $21.58(1.33)$ & $21.95(1.40)$ & $\mathrm{t}=0.982$ & $P=0.331$ \\
\hline APACHE II score(SD) ${ }^{\mathrm{a}}$ & $21.4 I(2.14)$ & $21.27(2.22)$ & $\mathrm{t}=0.23 \mathrm{I}$ & $P=0.818$ \\
\hline GCS score $n(\%)^{c}$ & & & \multirow{6}{*}{$\chi^{2}=6.430$} & \multirow{6}{*}{$P=0.16$} \\
\hline 4 & $4(14.8)$ & $3(I 1.5)$ & & \\
\hline 5 & $5(18.6)$ & $8(30.8)$ & & \\
\hline 6 & $12(44.4)$ & $4(15.4)$ & & \\
\hline 7 & $4(14.8)$ & $6(23.1)$ & & \\
\hline 8 & $2(7.4)$ & $5(19.2)$ & & \\
\hline
\end{tabular}

Notes: $P$ value $>0.05$ is nonsignificant; ${ }^{\mathrm{a}}$-test performed; ${ }^{\mathrm{b}} \mathrm{Chi}$-square performed; ${ }^{\mathrm{c}}$ Fisher exact test performed.

Abbreviations: BMI, body mass index; GCS, Glasgow Coma Scale/Score; APACHE II, Acute Physiology and Chronic Health Evaluation II. 
Table 2 Comparison of Nosocomial Infection and Adverse Events Between the Two Groups

\begin{tabular}{|l|l|l|l|l|}
\hline Outcome & Intervention Group $(\mathbf{n = 2 7})$ & Control Group $(\mathbf{n = 2 6})$ & $\chi^{\mathbf{2}}$ value & $\boldsymbol{P}$-value \\
\hline Nosocomial infection n (\%) & $5(18.5)$ & $13(50)$ & 5.853 & $P=0.016$ \\
\hline Adverse events n (\%) & $7(33.38)$ & $14(66.7)$ & 4.316 & $P=0.038$ \\
\hline
\end{tabular}

Notes: An adverse event was defined as one or more symptoms of diarrhea, or refeeding syndrome, or gastric retention; Nosocomial infection events: After 48 hours of hospitalization, all patients in the hospital were infected.Refeeding syndrome was defined to various manifestations related to metabolic abnormalities caused by re-intake (including oral intake, enteral or parenteral nutrition) after a long period of drought; Gastric retention was defined as anyone who vomits food that was ingested 4 to 6 hours before, or on an empty stomach for more than 8 hours, the residual amount in the stomach $>200 \mathrm{~mL}$; Diarrhea was defined as noninfectious bowel movements greater than five times per day and the excrement with a fluid characteristic; $P$ value $<0.05$ is significant; ${ }^{b}$ Chi-square performed.

in the early stage of critical illness, the stress response to injury will be disrupted. ${ }^{5}$ Patients during this period of time presents severe inflammation, insulin resistance, and intolerance to enteral feeding. ${ }^{6}$ Therefore, they believe that permissive underfeeding may be more beneficial in the acute phase of severe brain injury patients. ${ }^{7}$

In the 1980s, researchers revealed that the physiological characteristics of severe TBI patients in the acute phase were large amounts of catabolism, increased energy expenditure, severe pro-inflammatory reactions, insulin resistance, and increased AGI (acute gastrointestinal injury) risk. Some studies have unilaterally emphasized the need to provide enough calories for patients with severe $\mathrm{TBI}^{8}$ while the appearance of insulin resistance, gastrointestinal dysfunction, hyperglycemia on the nervous system, and other sequelae was caused by the lack of evaluation. ${ }^{9}$ After entering the ICU, patients with severe brain injury will be given corresponding treatments such as hypothermia, sedation, and mechanical ventilation. These treatment measures reduce the patient's resting energy expenditure by $10-30 \% .{ }^{10}$ Moreover, the use of tranquilizers and muscle relaxants further aggravates severe gastrointestinal dysfunction. This clinical study presented that patients in the intervention group experienced less gastrointestinal disorders, electrolyte imbalances, and high blood sugar compared to patients in the control group (Table 2). Therefore, blindly emphasizing adequate nutritional support in the acute phase of severe brain injury will only cause more complications, adversely affecting the prognosis of patients.

In the well-known PermiT test, Arabi et $\mathrm{al}^{11}$ compared permissive feeding (providing $40-60 \%$ of the target energy) with full feeding ( $70-100 \%$ of the target energy) for the impact of critically ill patients. The results of the study demonstrated that there was no difference in infection, ICU hospitalization, ICU mortality, or 90-day mortality between the two groups. However, these early lowcalorie nutritional supports can be observed by reviewing recent clinical trials of nutritional support. There are more or fewer deficiencies in the design of the treatment in clinical research. The Permit test only limits calories, not the intake of protein (the protein intake of both groups was $1.2-1.5 \mathrm{~g} / \mathrm{kg}$ per day). In contrast, there is no special selection for severe brain injury patients to undergo

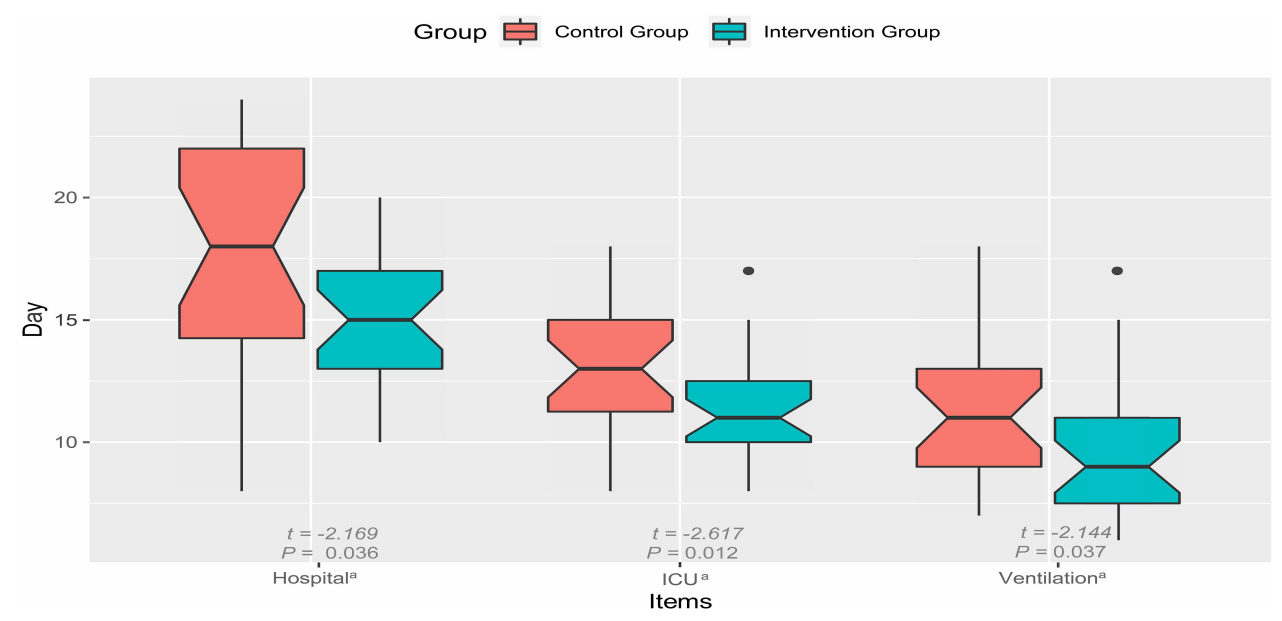

Figure I Comparison of total hospital stay, ICU stay, and mechanical ventilation time. Duration in the ICU or hospital or on a ventilator greater than 12 hours per day is counted as one day. ${ }^{a}$ t-test performed. $P$ value $<0.05$ is significant. 


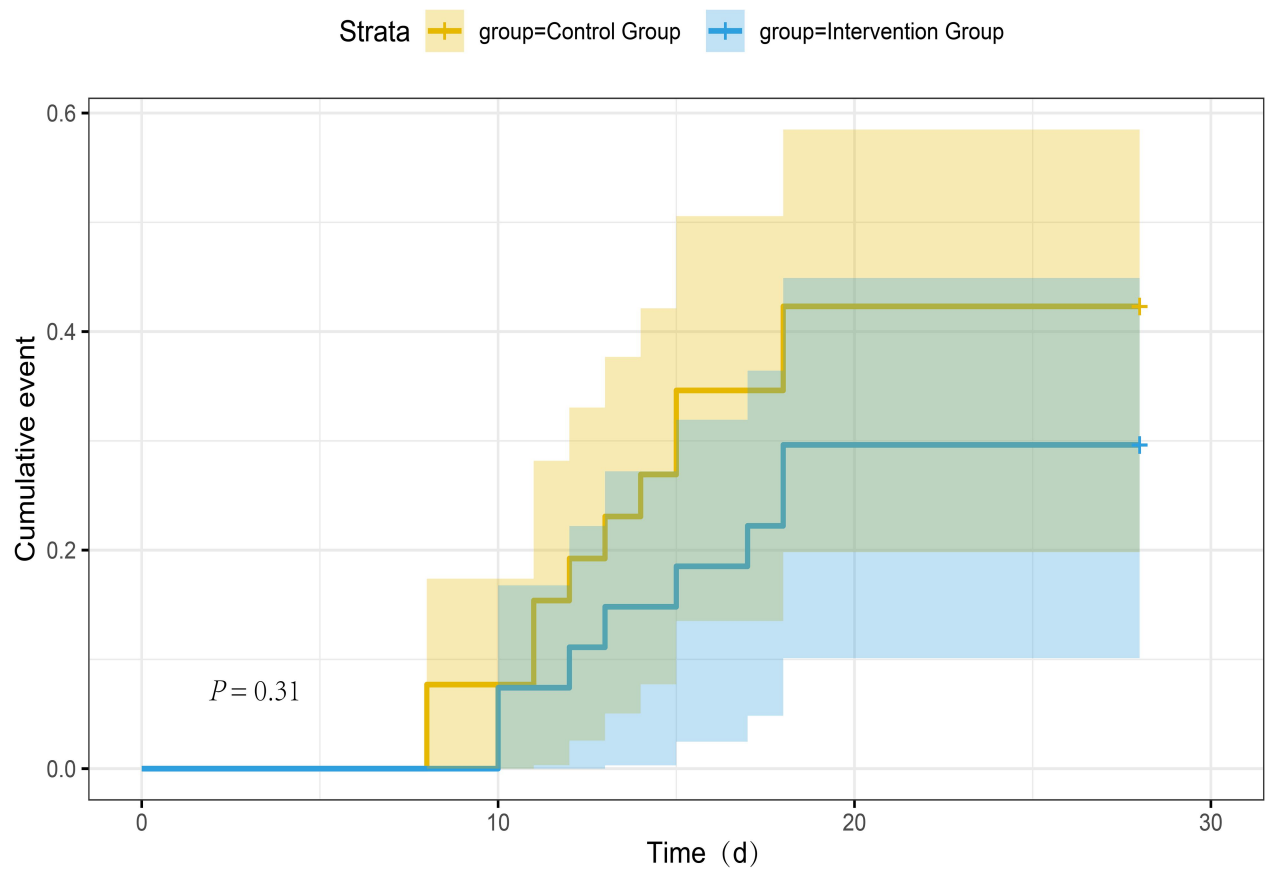

Figure 2 Comparison of 28-day mortality between the two groups.

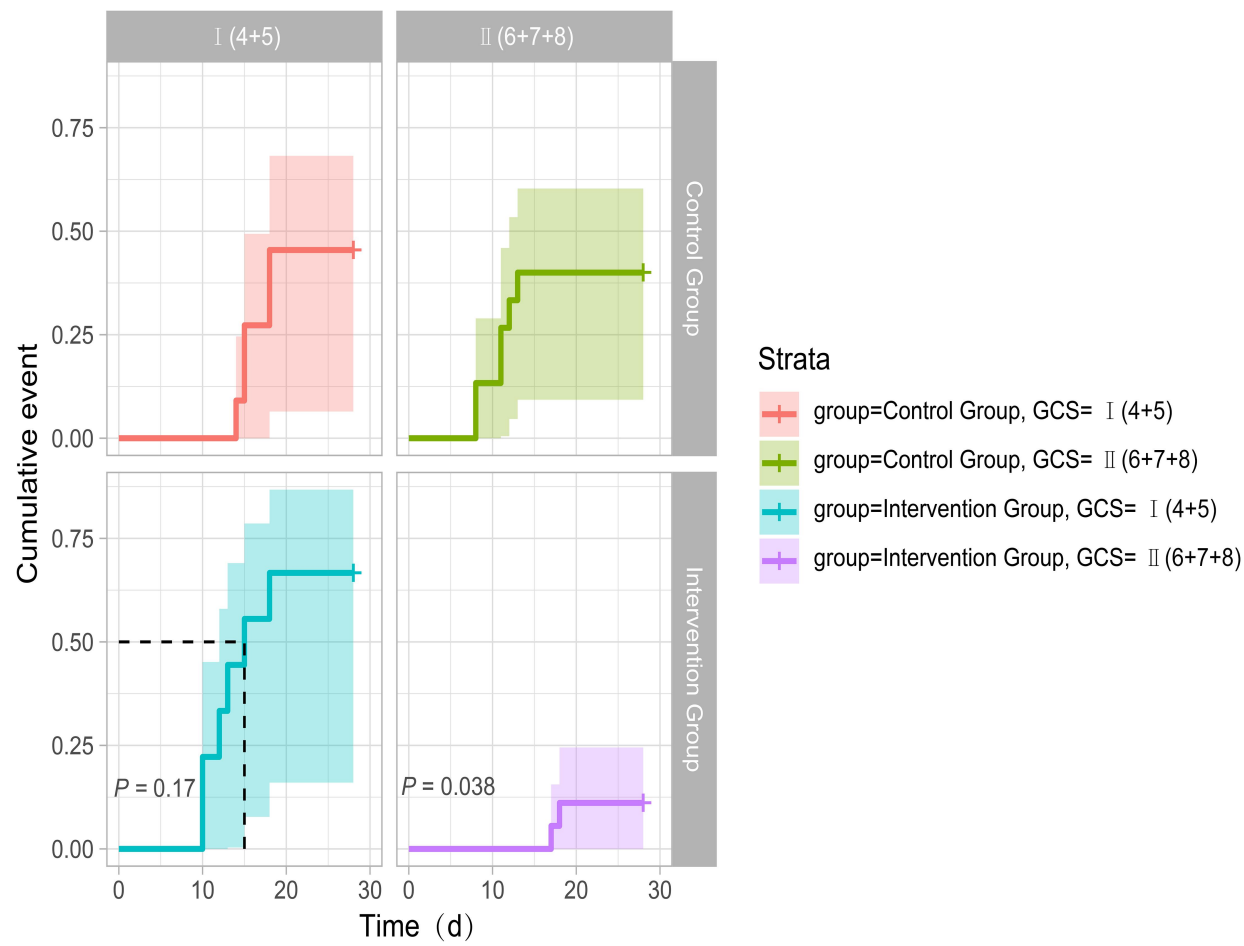

Figure 3 Subgroup analysis 28 -day mortality between the two groups based on GCS score.

intervention studies, though the EDEN trial ${ }^{12}$ (Early and Delayed Feeding Treatment of Acute Lung Injury or Acute Respiratory Distress Syndrome) studies have restricted calorie and protein intake. Therefore, the results of those clinical studies are worthy of further discussion.
During the development of severe TBI, the relationship between the body and nutrition becomes extremely complicated. In this clinical trial, the patients we enrolled suffered from a severe brain injury. The total amount of protein given to the observation group in the acute phase 
was $0.5-0.7 \mathrm{~g} /(\mathrm{kg} \cdot \mathrm{d})$, and the intake of glutamine was 0.3 $\mathrm{g} /(\mathrm{kg} \cdot \mathrm{d})$. Someone will definitely ask, why do we design the experimental program in this way? The answer is that glutamine is an indispensable nutrient in the human body. ${ }^{13}$ When a severe brain injury occurs, Glutamine in the body gradually decreases, and endogenous glutamine cannot meet the needs of the body under stress, aggravating metabolic disorders and making many severe patients have very poor clinical outcomes. ${ }^{14}$ Therefore, some researchers suggest that patients with severe brain injury should be given supplementary glutamine at an early stage. ${ }^{15-17}$

Studies have discovered that both glutamine and lowdose amino acids have the potential to activate autophagy. ${ }^{18}$ Besides, the effect of autophagy explains that preserving (or minimizing) early nutrition may be more beneficial to critically ill patients. ${ }^{19}$ Recent observational studies have revealed ${ }^{20,21}$ that providing nutritional support rich in glutamine, either alone ${ }^{22}$ or combined with probiotics $^{23}$ or alanine, ${ }^{24}$ can reduce hospitalization time and infection of moderate to severe traumatic brain injury. In the CALORIES ${ }^{25}$ and NUTRIREA- $2^{6}$ trials, lower calories and protein intake during the first week of ICU stay exhibited better clinical outcomes. Stroud ${ }^{26}$ compared the effects of different protein intakes in the treatment of protein-energy malnutrition, concluding that high-protein intakes are dangerous.

The National Institute of Health and Clinical Optimization (NICE) once recommended that nutritional support for patients with severe illness or injury start with an estimated $50 \%$ of the target energy and protein requirements. ${ }^{26}$ In clinical trials, we discovered that time in ICU, hospital stay, and ventilator time of the patients in the intervention group were significantly reduced (Figure 1). Therefore, we recommend that patients with severe brain injury should be given low-protein, hypocaloric nutrition supplemental glutamine at an early stage.

Since glutamine is administered parenterally, only single-blind experiments can be performed. The included critically ill patients were given enteral + parenteral nutrition support within 24 hours after admission. After nutritional support was provided, patients in the intervention group had a slight improvement in the primary endpoint (28-day mortality), though there was no statistical difference (Figure 2). However, the 28-day mortality of the two groups could not be simply compared. If we extend the use time of glutamine to 14 days according the ESPEN guideline, maybe there will be a statistical difference between the two groups.Regarding secondary endpoint, this clinical trial provided a reliable and accurate estimate of the infection rate, including catheter-related infections. The results showed that there were significant differences in nosocomial infection events between the two groups.Perhaps due to the reduction of nosocomial infections, patients in the intervention group also significantly reduced the time spent on ICU and mechanical ventilation (Table 2). From another perspective, the use of glutamine-enhanced lowprotein low-calorie nutritional support in this clinical trial did not cause harm to critically ill patients with high nutritional risk. The patient's mechanical ventilation time was significantly reduced compared to the control group; the results were similar to the results of Arabi. ${ }^{27}$ It was observed that the increase in calorie intake was independently related to the significant extension of mechanical ventilation time. The clinical studies of $\mathrm{EPaNIC}^{28}$ and PEPaNIC $^{29}$ indicated that the early extraintestinal damage was caused by the increase in the dose of amino acids instead of other macronutrients in the recent secondary statistical analysis. The possible reason was that the high dose of amino acids strongly inhibited autophagy. In critical illness, the interaction between autophagy and immune response and inflammation is complex and has not been fully elucidated.

We performed a subgroup survival analysis and comparison of the included patients according to the GCS score, revealing that the patients with higher GCS scores received inadequate permissive feeding of low protein with glutamine compared to the control group; the 28day mortality was significantly different (Figure 3). The possible reasons for this phenomenon need to be further explored.

Although the subgroup analysis is conducted according to the GCS score, the sample size between the two groups is still small, and the statistical power is poor. The clinical trial is a single-blind trial. It is safe. Meanwhile, it is inevitable that we will have some biases when implementing the treatment plan and evaluating the efficacy. This is the shortcoming of this clinical trial.

\section{Conclusion}

Although in this clinical trial, low-protein, hypocaloric nutritional support with appropriate supplemental glutamine given to critically ill patients with STBS in the acute phase did not decrease the patient's 28-day mortality, the subgroup analysis exhibited some good effects on the results, and the secondary endpoints were significantly 
improved. Therefore, whether the low-protein and hypocaloric nutritional support treatment with glutamine in the acute phase has a relatively good effect on the outcome of critically ill patients with STBS deserves further investigation.

\section{Data Sharing Statement}

All necessary information about this study was presented in the manuscript. Owing to the respect and protection of our patients' privacy, the raw data regarding our patients are not publicly available, but it is available from the corresponding authors upon reasonable request.

\section{Disclosure}

The authors declare no conflicts of interest.

\section{References}

1. Taylor CA, Bell JM, Breiding MJ, Xu L. Traumatic brain injury-related emergency department visits, hospitalizations, and deaths - United States, 2007 and 2013. MMWR Surveill Summ. 2017;66(9):1-16. doi:10.15585/mmwr.ss6609a1

2. Singer P, Blaser AR, Berger MM, et al. ESPEN guideline on clinical nutrition in the intensive care unit. Clin Nutr. 2019;38(1):48-79. doi:10.1016/j.clnu.2018.08.037

3. Carney N, Totten AM, O'Reilly C, et al. Guidelines for the management of severe traumatic brain injury, fourth edition. Neurosurgery. 2017;80(1):6-15. doi:10.1227/neu.0000000000001432

4. McClave SA, Heyland DK. The physiologic response and associated clinical benefits from provision of early enteral nutrition. Nutr Clin Pract. 2009;24(3):305-315. doi:10.1177/0884533609335176

5. Marik PE. Is early starvation beneficial for the critically ill patient? Curr Opin Clin Nutr Metab Care. 2016;19(2):155-160. doi:10.1097/ mco.0000000000000256

6. Reignier J, Boisramé-Helms J, Brisard L, et al. Enteral versus parenteral early nutrition in ventilated adults with shock: a randomised, controlled, multicentre, open-label, parallel-group study (NUTRIREA-2). Lancet. 2018;391(10116):133-143. doi:10. 1016/s0140-6736(17)32146-3

7. Allingstrup MJ, Kondrup J, Wiis J, et al. Early goal-directed nutrition versus standard of care in adult intensive care patients: the single-centre, randomised, outcome assessor-blinded EAT-ICU trial. Intensive Care Med. 2017;43(11):1637-1647. doi:10.1007/s00134017-4880-3

8. Abdullah MI, Ahmad A, Tarek SW, et al. Determination of calorie and protein intake among acute and sub-acute traumatic brain injury patients. Chin J Traumatol = Zhonghua Chuang Shang Za Zhi. 2020;23(5):290-294. doi:10.1016/j.cjtee.2020.04.004

9. Quintard H, Ichai C. Nutritional and metabolic supplementation for the injured brain: an update. Curr Opin Crit Care. 2019;25(2): 126-131. doi:10.1097/mcc.0000000000000588

10. Frankenfield DC, Ashcraft CM. Description and prediction of resting metabolic rate after stroke and traumatic brain injury. Nutrition 2012;28(9):906-911. doi:10.1016/j.nut.2011.12.008

11. Arabi YM, Aldawood AS, Solaiman O. Permissive underfeeding or standard enteral feeding in critically ill adults. $N$ Engl $J$ Med. 2015;373(12):1175-1176. doi:10.1056/NEJMx150028

12. Heart TN. Initial trophic vs full enteral feeding in patients with acute lung injury: the EDEN randomized trial. JAMA. 2012;307(8): 795-803. doi:10.1001/jama.2012.137
13. Sandoval CP. Glutamine supplementation for critically ill adults: a summary of findings from the cochrane library with implications for critical care nursing. Crit Care Nurse. 2015;35(6):71-72. doi: $10.4037 / \mathrm{ccn} 2015220$

14. Leite JS, Raizel R, Hypólito TM, Rosa TD, Cruzat VF, Tirapegui J. l-glutamine and l-alanine supplementation increase glutamine-glutathione axis and muscle HSP-27 in rats trained using a progressive high-intensity resistance exercise. Appl Physiol Nutr Metab. 2016;41(8):842-849. doi:10.1139/apnm-2016-0049

15. Moe-Byrne T, Brown JV, McGuire W. Glutamine supplementation to prevent morbidity and mortality in preterm infants. Cochrane Database Syst Rev. 2016;4(4):Cd001457. doi:10.1002/14651858.CD001457.pub6

16. Shariatpanahi ZV, Eslamian G, Ardehali SH, Baghestani AR. Effects of early enteral glutamine supplementation on intestinal permeability in critically ill patients. Indian J Crit Care Med. 2019;23(8):356-362. doi:10.5005/jp-journals-10071-23218

17. Abe T, Hosoi T, Kawai R, et al. Perioperative enteral supplementation with glutamine, fiber, and oligosaccharide reduces early postoperative surgical stress following esophagectomy for esophageal cancer. Esophagus. 2019;16(1):63-70. doi:10.1007/s10388-018-0630-z

18. Wernerman J, Christopher KB, Annane D, et al. Metabolic support in the critically ill: a consensus of 19. Crit Care. 2019;23(1):318. doi:10.1186/s13054-019-2597-0

19. Van Dyck L, Casaer MP, Gunst J. Autophagy and its implications against early full nutrition support in critical illness. Nutr Clin Pract. 2018;33(3):339-347. doi:10.1002/ncp.10084

20. Stehle P, Ellger B, Kojic D, et al. Glutamine dipeptide-supplemented parenteral nutrition improves the clinical outcomes of critically ill patients: a systematic evaluation of randomised controlled trials. Clin Nutr ESPEN. 2017;17:75-85. doi:10.1016/j.clnesp.2016.09.007

21. Apostolopoulou A, Haidich AB, Kofina K, et al. Effects of glutamine supplementation on critically ill patients: focus on efficacy and safety. An overview of systematic reviews. Nutrition. 2020;78:110960. doi:10.1016/j.nut.2020.110960

22. Nägeli M, Fasshauer M, Sommerfeld J, Fendel A, Brandi G, Stover JF. Prolonged continuous intravenous infusion of the dipeptide L-alanineL-glutamine significantly increases plasma glutamine and alanine without elevating brain glutamate in patients with severe traumatic brain injury. Crit Care. 2014;18(4):R139. doi:10.1186/cc13962

23. Juang P, Fish DN, Jung R, MacLaren R. Enteral glutamine supplementation in critically ill patients with burn injuries: a retrospective case-control evaluation. Pharmacotherapy. 2007;27(1):11-19. doi:10. 1592/phco.27.1.11

24. Zeng J, Zhao XY, Huang Q, Wang ER. [Effects of glutamine-enriched enteral nutrition on nutritional status and prognosis of patients with severe head injury]. Zhonghua Shao Shang Za Zhi. 2009;25(5):335-338. Chinese.

25. Casaer MP, Mesotten D, Hermans G, et al. Early versus late parenteral nutrition in critically ill adults. $N$ Engl J Med. 2011;365(6):506-517. doi:10.1056/NEJMoa1102662

26. Stroud M. Protein and the critically ill; do we know what to give? Proc Nutr Soc. 2007;66(3):378-383. doi:10.1017/s0029665107005642

27. Arabi YM, Haddad SH, Tamim HM, et al. Near-target caloric intake in critically ill medical-surgical patients is associated with adverse outcomes. JPEN J Parenter Enteral Nutr. 2010;34(3):280-288. doi:10.1177/0148607109353439

28. Casaer MP, Wilmer A, Hermans G, Wouters PJ, Mesotten D, Van den Berghe G. Role of disease and macronutrient dose in the randomized controlled EPaNIC trial: a post hoc analysis. Am J Respir Crit Care Med. 2013;187(3):247-255. doi:10.1164/rccm.201206-0999OC

29. Vanhorebeek I, Verbruggen S, Casaer MP, et al. Effect of early supplemental parenteral nutrition in the paediatric ICU: a preplanned observational study of post-randomisation treatments in the PEPaNIC trial. Lancet Respir Med. 2017;5(6):475-483. doi:10. 1016/s2213-2600(17)30186-8 


\section{Publish your work in this journal}

Neuropsychiatric Disease and Treatment is an international, peerreviewed journal of clinical therapeutics and pharmacology focusing on concise rapid reporting of clinical or pre-clinical studies on a range of neuropsychiatric and neurological disorders. This journal is indexed on PubMed Central, the 'PsycINFO' database and CAS, and is the official journal of The International Neuropsychiatric Association (INA). The manuscript management system is completely online and includes a very quick and fair peer-review system, which is all easy to use. Visit http://www.dovepress.com/testimonials.php to read real quotes from published authors. 